\title{
An Approach for Understanding and Promoting Coal Mine Safety by Exploring Coal Mine Risk Network
}

\author{
Yongliang Deng, ${ }^{1,2}$ Liangliang Song, ${ }^{3}$ Zhipeng Zhou, ${ }^{4}$ and Ping Liu ${ }^{3,5}$ \\ ${ }^{1}$ State Key Laboratory for Geomechanics \& Deep Underground Engineering, China University of Mining and Technology, \\ Xuzhou 22116, China \\ ${ }^{2}$ School of Mechanics and Civil Engineering, China University of Mining and Technology, Xuzhou 221116, China \\ ${ }^{3}$ School of Civil Engineering, Southeast University, Nanjing 210096, China \\ ${ }^{4}$ College of Economic and Management, Nanjing University of Aeronautics and Astronautics, Nanjing 210016, China \\ ${ }^{5}$ School of Civil Engineering, Lanzhou University of Technology, Lanzhou 730050, China \\ Correspondence should be addressed to Liangliang Song; 230129183@seu.edu.cn
}

Received 26 May 2017; Accepted 22 August 2017; Published 12 October 2017

Academic Editor: Gerard Olivar

Copyright @ 2017 Yongliang Deng et al. This is an open access article distributed under the Creative Commons Attribution License, which permits unrestricted use, distribution, and reproduction in any medium, provided the original work is properly cited.

\begin{abstract}
Capturing the interrelations among risks is essential to thoroughly understand and promote coal mining safety. From this standpoint, 105 risks and 135 interrelations among risks had been identified from 126 typical accidents, which were also the foundation of constructing coal mine risk network (CMRN). Based on the complex network theory and Pajek, six parameters (i.e., network diameter, network density, average path length, degree, betweenness, and clustering coefficient) were employed to reveal the topological properties of CMRN. As indicated by the results, CMRN possesses scale-free network property because its cumulative degree distribution obeys power-law distribution. This means that CMRN is robust to random hazard and vulnerable to deliberate attack. CMRN is also a small-world network due to its relatively small average path length as well as high clustering coefficient, implying that accident propagation in CMRN is faster than regular network. Furthermore, the effect of risk control is explored. According to the result, it shows that roof collapse, fire, and gas concentration exceeding limit refer to three most valuable targets for risk control among all the risks. This study will help offer recommendations and proposals for making beforehand strategies that can restrain original risks and reduce accidents.
\end{abstract}

\section{Introduction}

China is the largest producer and consumer of coal in the world, from which it has derived about $65 \%$ of its energy over the past sixty years [1]. In China, more than $90 \%$ of fossil energy reserves are coal. That is to say, the energy consumption structure of energy, which relies mainly on coal, cannot be changed within quite a long time. Also, this standpoint can be validated by China's National Energy Development Strategy Plan (2014-2020) and 13th Five-Year Plan (2016-2020). In 2015, China's coal output was estimated to be 3.747 billion tons, accounting for $47 \%$ of the total in the world (The State Administration of Coal Mine Safety, 2015). According to British Petroleum (BP) Statistical Review of World Energy 2016, the countries whose coal production is larger than 40 million tons can be shown in Figure 1.
Coal mining refers to one of the most hazardous industries worldwide [2-4]. Moreover, coal mine enterprises have to encounter various hazards regarding special geological condition [3]. In the process of coal mining, numerous hazards have the potential to trigger accidents frequently, such as rock stresses, harmful gases, humidity, high temperatures, coal and silica dust, and specialized equipment [5]. Worse still, the intensity and frequency of these hazards could result in extremely serious consequences for human health and life [6]. Coal mine accidents will considerably bring about injuries, casualties, and loss of major assets of enterprise. In China, coal mine accident suffers heavy losses every year. According to statistics, approximately $70 \%$ of the coal mine casualties worldwide are estimated to occur in China [7]. 6995 coal workers were killed in various accidents in 2002, which is the maximum record in a single year. 


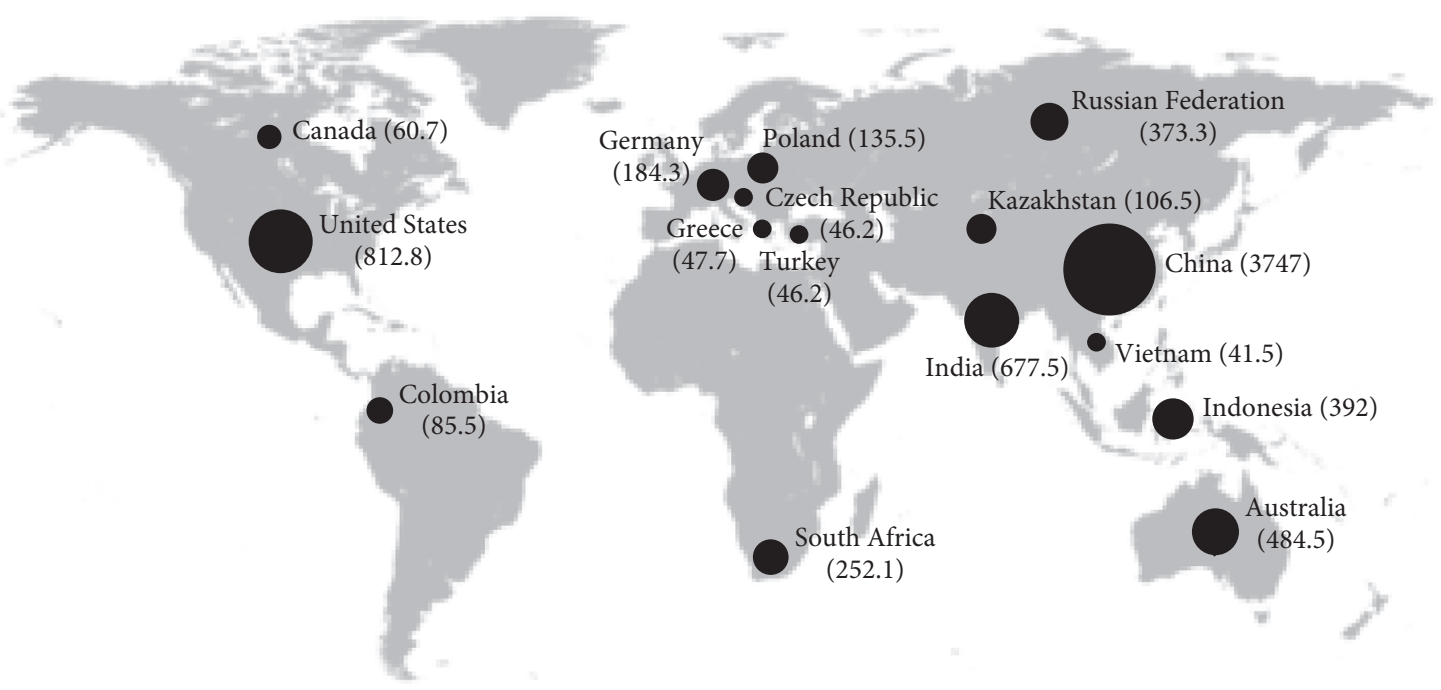

FIGURE 1: The coal production distributed by country in 2015 (one hundred million tons).

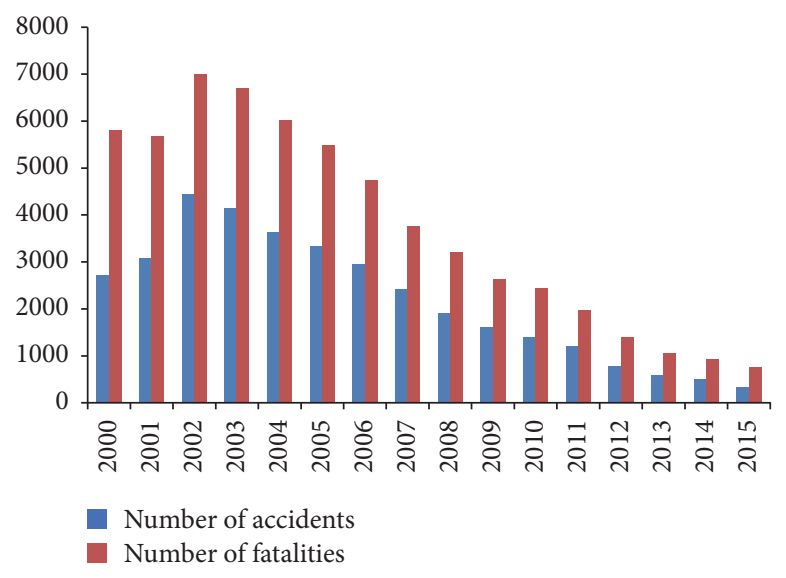

Figure 2: China's number of accidents and fatalities in coal mine from 2000 to 2015.

Then, it decreased year by year, as shown in Figure 2 (data source: State Administration of Coal Mine Safety). Although the practical situation seemingly gets better and better, still numerous accidents occurred every year in China. All in all, safety management in coal production is still quite critical and serious due to harsh production conditions as well as complicated production processes.

A more valuable process to improve safety performance is to learn from the failure experiences of previous accidents [8]. Accident analysis is a powerful approach for preventing or eliminating similar hazards, risks, and accidents $[9,10]$. Indeed, the existing studies often focus on one type of coal mining accident, or statistical analysis of accident in an area or country, while multiple interrelations among verified accidents are usually neglected. In industrial safety research, it is generally acknowledged that the accident is not caused by a single error or fault, but by the confluence of a sequence of hazard, risk, and accident [11]. Moreover, an occurred accident will possibly incur a sequence of the following accidents [12]. Accident chain exists in most of the coal mine accidents, which indicates the actual existence of risk network. These interactions among risks form a coal mine risk network (CMRN) which would bring about a big issue for the coal mine safety. Therefore, capturing the complexity of CMRN is both essential and beneficial to improve safety performance in coal mining.

The structure of this paper can be listed as follows. Section 2 presents a literature review of coal mine safety, and Section 3 elaborates the methodology, including an analytical framework, data collection and analysis, and network modeling. In Section 4, Pajek is employed to help explore CMRN (including network basic quantities metric and network property) and measure the effect of risk control. In Section 5, the potential contributions, limitations, and risk control methods are discussed. Lastly, the conclusions are drawn in Section 6.

\section{Literature Review}

Coal mine provides essential energy for supporting highspeed development of Chinese economy and society. Multiple 
TABLE 1: Summary of previous study in coal mine.

\begin{tabular}{|c|c|c|}
\hline Theme & References & Objective \\
\hline Supervision and regulation & {$[13-18]$} & $\begin{array}{l}\text { Exploring complexity and ineffectiveness of regulation; analyzing rent-seeking } \\
\text { mechanism, behavior, policy, and tax; identifying tendency of coal mine accidents } \\
\text { and characteristics of human factors. }\end{array}$ \\
\hline Risk management & {$[19-24]$} & $\begin{array}{l}\text { Predicting the expected risk levels by using decomposition technique in time series } \\
\text { analysis; analyzing and optimizing the risk management system; using public } \\
\text { communication system to monitor unsafe behavior in real time; reducing the effects } \\
\text { of coal mining on social and ecological exploitation; constructing potential hazards } \\
\text { database in an underground mine; evaluating the reliability of human safety barrier } \\
\text { in coal mine emergency evacuation; identifying the risk factors and evaluating the } \\
\text { safety control capability. }\end{array}$ \\
\hline Risk evaluation & {$[25-31]$} & $\begin{array}{l}\text { Assessing the roof fall risk during retreat mining in room and pillar coal mines; } \\
\text { evaluating explosion risk in underground coal mines; developing a comprehensive } \\
\text { model for coal mine safety; using fuzzy set theory to assess the risk of mining } \\
\text { equipment failure; assessing pot-hole subsidence risk in coal mine; using risk } \\
\text { performance indicators to analyze coal mine accidents. }\end{array}$ \\
\hline $\begin{array}{l}\text { Monitoring and controlling } \\
\text { technologies }\end{array}$ & [32-36] & $\begin{array}{l}\text { Employing internet of things (IOT) and cloud computing (CC) to monitor mine } \\
\text { safety based on prealarm system; using wireless sensor network (WSN) to monitor } \\
\text { the temperature, humidity, gas, and status of smoke in underground mine; } \\
\text { establishing a Web of Things-based remote monitoring system for coal mine safety; } \\
\text { employing cable monitoring system (CMS) and the WSN to build an integrated } \\
\text { environment monitoring system for underground coal mine; using iris } \\
\text { identification and radio frequency identification (RFID) technique to improve } \\
\text { safety management system. }\end{array}$ \\
\hline
\end{tabular}

studies have been carried out by worldwide researchers to improve the safety performance. The research topics mainly focus on supervision and regulation, risk management, evaluation, monitoring, and controlling technologies, which is shown in Table 1.

Supervision and regulation refer to two crucial influence factors in the coal mining. Before 2000, ineffective implementation of laws and regulations increases the difficulty for Chinese government to inspect actual situation of coal mine safety [13]. To promote coal mine safety, a variety of effective countermeasures, such as enhancing safety legislation and establishing independent coal mine safety monitoring system, were executed. These improvements in regulatory regime make a great contribution [14]. However, the interrelations between coal mine enterprises and supervision departments are complex and subtle. Rent-seeking exists widely in China's coal mine supervision, which is a huge obstacle to the further development of coal mining industry. The existing researches on rent-seeking mainly focus on rentseeking behavior, policy, and tax [15-17]. In the rent-seeking scenario, Chen et al. [18] indicated that each level of the department had an intensity threshold above which coal mine accidents occurred.

The effective risk management is the fundamental guarantee of coal mine safety production based on various theories and methods. Sari et al. [19] developed a stochastic model to predict the number of accidents according to the randomness in the occurrence of accidents. Qing-gui et al. [20] constructed a system to supervise unsafe behavior, release early warning information, and improve controlling measures in coal mine. Based on case studies, Kowalska [21] identified and assessed the risk sources. As suggested by the results, it is necessary to undertake anticipatory activities aiming at reducing environmental and social risks during the colliery liquidation. Badri et al. [22] studied risk management in mining projects based on analytic hierarchy process method, and the results show the importance of considering occupational health and safety (OHS) in the process of coal mining. Wang et al. [23] put forward an analytical framework to analyze human error risk in the emergency evacuation from three perspectives, including organization level, group level, and individual level. Besides, Liu and Li [24] constructed a back propagation (BP) neural network to explore influence factors in coal mine safety.

The evaluation of hazards and risks has attracted much attention of multiple practitioners and researchers due to their serious consequences. These hazards and risks could be divided into three types, including "natural, technical, and human." Ghasemi et al. [25] developed a risk evaluation model and various possible risks are evaluated in Iran Tabas central mine. Pejic et al. [26] proposed a risk assessment tool to determine the risk of explosion of any work processes or activities in the underground coal mine. Also, the methodology can decide whether the proposal investments are well-justified or not for improving safety. Bahri Najafi et al. [27] proposed an artificial neural network model to predict the out-of-seam dilution. Based on uncertain random variables, Chen et al. [28] developed a practical evaluation model for coal mine safety based on uncertain random variables. According to fuzzy set theory, Petrović et al. [29] presented a risk evaluation model to evaluate the failures of electromechanical equipment. Lokhande et al. [30] came up with a risk evaluation approach based on the identified critical parameters, including depth to height of extraction 


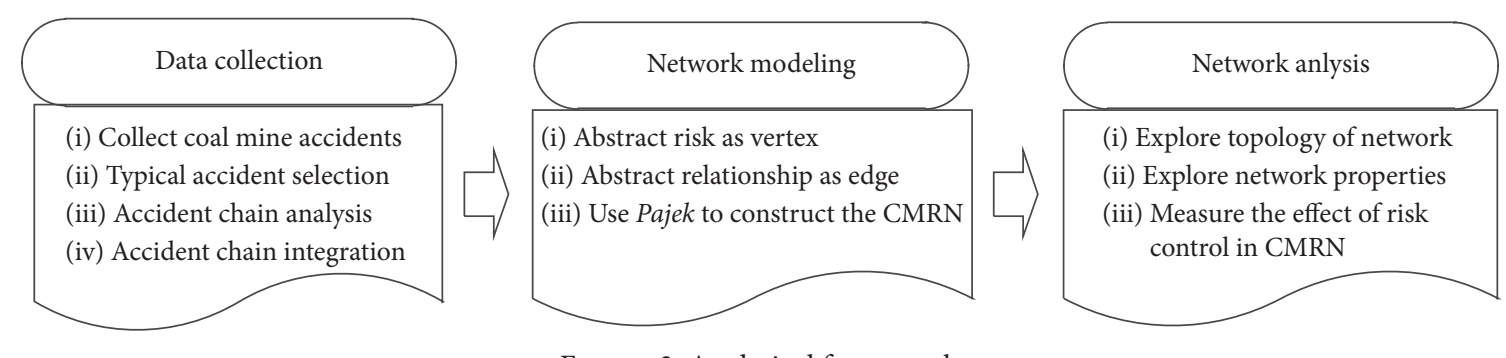

FIgURe 3: Analytical framework.

TABLE 2: Two examples of the stored accidents.

\begin{tabular}{|c|c|c|c|c|c|c|}
\hline Number & Time & City & Type & Description & Death & Loss \\
\hline 28 & 2003.10 .21 & Wuhai & $\begin{array}{l}\text { Coal dust } \\
\text { explosion }\end{array}$ & $\begin{array}{l}\text { Three blasters violated job regulations and } \\
\text { implemented blasting without any safety precautions. } \\
\text { Unfortunately, the blasting gave rise to naked light, } \\
\text { and then the gas was lighted and began burning. As a } \\
\text { result, the coal dust explosion happened. }\end{array}$ & 6 & $\begin{array}{c}120 \text { thousand } \\
\text { dollars }\end{array}$ \\
\hline 92 & 2004.2 .23 & Jixi & $\begin{array}{c}\text { Gas } \\
\text { explosion }\end{array}$ & $\begin{array}{c}\text { Due to inadequate ventilation, the gas concentration } \\
\text { exceeded the threshold. Meanwhile, a miner } \\
\text { optionally disassembled his lamp, which triggered } \\
\text { electric spark, and then the spark caused gas } \\
\text { explosion. }\end{array}$ & 37 & $\begin{array}{c}370 \text { thousand } \\
\text { dollars }\end{array}$ \\
\hline
\end{tabular}

ratio, rock to soil ratio, brittleness index of rock, and rock density. Spada and Burgherr [31] analyzed the accident data in energy-related severe accidents database and suggested a nonsignificant decreasing tendency for Turkey as well as a significant one for USA.

Some new technologies, which are effective and powerful tools for improving safety performance, have been applied in coal mine. Sun et al. [32] accomplished a monitoring and prealarm system based on cloud computing (CC) and Internet of things (IOT). What is more, Dange and Patil [33] designed a wireless sensor network (WSN) based on MSP430 controller for monitoring smoke, gas, temperature, and humidity in coal mine. Based on wireless sensor network and controller area network (CAN), Bo et al. [34] proposed a remote monitoring system, which was tested in different remote monitoring scenarios. Zhang et al. [35] proposed an integrated environment monitoring system that takes full advantage of cable monitoring system (CMS) in combination with wireless sensor network (WSN). Xu et al. [36] put forward an improved safety management system based on several modern identification and communication techniques, including iris identification, radio frequency identification (RFID), computer network, and database technique.

\section{Methodology}

3.1. Analytical Framework. An analytical framework is proposed to conduct the in-depth analysis of coal mine accident, as presented in Figure 3. It is a step-by-step procedure consisting of three main modules. At first, the coal mine accidents are collected from literature and media, such as the website of State Administration of Coal Mine Safety. Then, typical accidents are selected as the data to analyze accident chains. After that, the accident chains will be integrated as a global network. In the second stage, the risk is abstracted as vertex, and meanwhile, the interrelation is abstracted as edge. Also, the software Pajek is employed to establish the coal mine risk network (CMRN). In the third stage, the topology of CMRN is analyzed and network properties are identified according to the network theory. Then, the effect of risk control in CMRN is calculated. According to the research result, the discussions and suggestions are provided to promote safety management in coal mine production.

3.2. Data Collection and Analysis. The data of historical coal mining accidents is used for risk analysis. There are several ways to collect accident cases, such as government, enterprise, literature, and media. In this study, the accidents are collected from literature and media. A coal mine accident database (CMAD), which records the detailed information of accident (including time, position, type, process, death, and losses), is established based on Microsoft Access 2010. Although hundreds of accidents have occurred in China over the past few years, the information of many accidents, especially the process of accident, is unclear. In the end, 176 accidents with exhaustive information are collected. Among these detailed accidents, some accident chains are unobvious, while some happen suddenly and unexpectedly without accident chain. These accidents are not considered in this research. Besides, since some accidents are exactly similar to the rest of the typical accidents, thus there is no need to analyze the repeating accidents. In the end, 126 typical accidents, including all types of coal mining accidents, are recorded in $\mathrm{CMAD}$, and they are selected to conduct accident chain analysis for establishing the risk network model. Two examples of stored accidents can be illustrated in Table 2 .

Although these accidents are selective, almost all kinds of accidents have been included. Also, there are no biases in the 


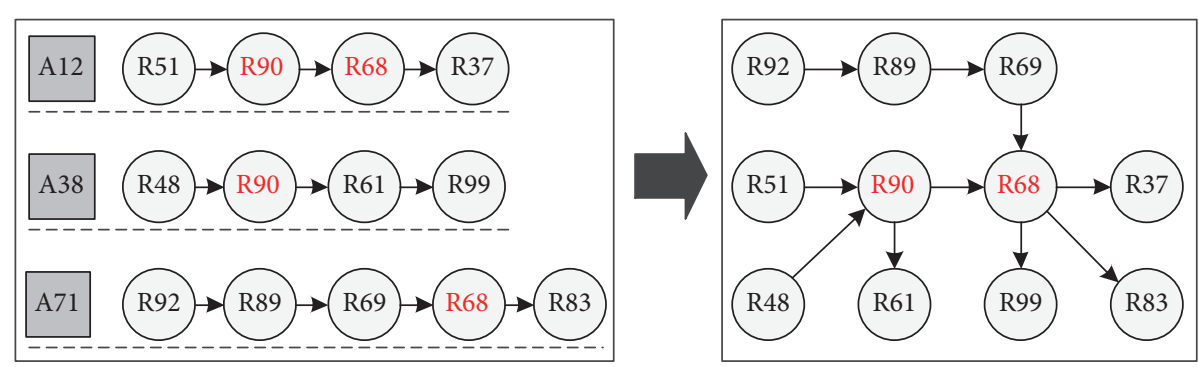

FIgURE 4: The formative process of CMRN.

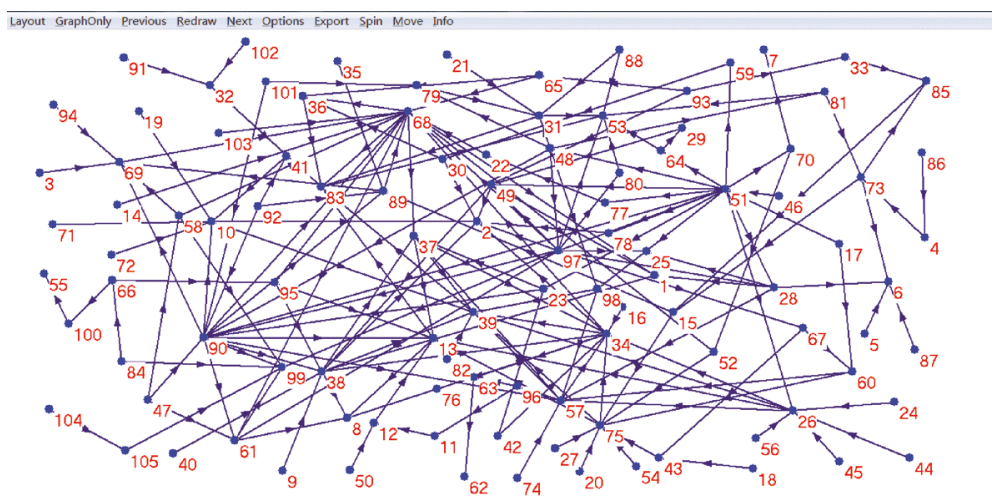

Figure 5: The coal mine risk network model in Pajek.

selection process. From the perspective of person, machine, environment, management, and technology, the accident chains in these accidents are identified and expatiated in Table 3. Most of the accidents have one accident chain, while some have two, such as accidents 41 and 75 . As a result, a total of 135 accident chains are obtained from 126 cases.

3.3. Network Modeling. Multiple risks simultaneously appear in different accidents, indicating that the risk is correlated with others. It is essential to identify the risks and interrelations among them so as to establish CMRN. Through statistics, a total of 105 risks and 194 interrelations are obtained from 135 accident chains. Moreover, the vertex number and its type are expatiated in Table 4. After this study abstracts risk as vertex and interrelation as edge, different risks can be connected by these common vertexes into a global network. For a better explanation, accidents 12,38 , and 71 are taken as an example to illustrate the process of network modeling, as depicted in Figure 4. From the risks identification in accidents 12,38 , and 71 , it can be seen that there are two same vertexes shown in red color, including R90 and R68. Through this method, the network can be established based on these common risks. Furthermore, software Pajek is employed to establish coal mine risk network (CMRN), as shown in Figure 5.

\section{Results}

4.1. Network Basic Quantities Metric. With the continuous development of complex network theory, the statistical indexes of network structure have obtained a lot of achievements, which are also the basis of statistical description of various topological characteristics. Compared with visual section, the calculation is much more precise and concise in exploring network [37]. This study uses several typical indexes to explore the properties of CMRN, including network diameter, network density, average path length, degree, betweenness, and clustering coefficient. These topological indexes are calculated by Pajek.

4.1.1. Network Diameter. The network diameter is defined as the maximum path length in the network, which can reflect the size of a network. The network diameter in CMRN is 7, which is from poor maintenance (vertex 64) to water leaking (vertex 99). This path is as follows: poor maintenance (vertex 64) causes electrified device failure (vertex 29), electrified device failure (vertex 29) triggers inadequate ventilation (vertex 49), inadequate ventilation (vertex 49) makes gas concentration exceed limit (vertex 38), gas concentration exceeding limit (vertex 38) incurs gas burning (vertex 37), gas burning (vertex 37) sparks off fire (vertex 34), fire (vertex 34) induces roof collapse (vertex 68), roof collapse (vertex 68) leads to penetration into goaf (vertex 61), and penetration into goaf (vertex 61) brings about water leaking (vertex 99). Although these risks may not occur simultaneity in a single accident, it can deeply reflect the process of risk spread. The spread rule of risk is conductive to developing prevention and control strategies for the risk control.

4.1.2. Network Density. Network density is used to describe the degree of affinity between the vertexes in a network from 
TABle 3: Accident chain analysis.

\begin{tabular}{|c|c|}
\hline Number & Accident chains \\
\hline 1 & Smoking $\rightarrow$ naked light $\rightarrow$ fire $\rightarrow$ suffocation \\
\hline 2 & Unreasonable blasting $\rightarrow$ gas concentration exceeding limit $\rightarrow$ gas burning $\rightarrow$ fire \\
\hline 3 & $\begin{array}{l}\text { Air blower failure } \rightarrow \text { inadequate ventilation } \rightarrow \text { gas concentration exceeding limit } \rightarrow \text { gas burning } \rightarrow \text { fire } \rightarrow \text { gas } \\
\text { explosion }\end{array}$ \\
\hline 4 & Electric leakage $\rightarrow$ gas burning $\rightarrow$ gas explosion \\
\hline 5 & Coal and gas outburst $\rightarrow$ suffocation \\
\hline 6 & Electrical failures $\rightarrow$ air blower failure $\rightarrow$ inadequate ventilation $\rightarrow$ Gas concentration exceeding limit $\rightarrow$ fire \\
\hline 7 & Violation operation $\rightarrow$ inadequate ventilation $\rightarrow$ gas concentration exceeding limit $\rightarrow$ gas explosion \\
\hline 8 & Inadequate ventilation $\rightarrow$ gas concentration exceeding limit $\rightarrow$ suffocation \\
\hline 9 & Violation operation $\rightarrow$ suffocation \\
\hline 10 & Management negligence $\rightarrow$ violation weld $\rightarrow$ gas explosion \\
\hline 11 & Management negligence $\rightarrow$ inadequate ventilation $\rightarrow$ suffocation \\
\hline 12 & Management negligence $\rightarrow$ unreasonable blasting $\rightarrow$ roof collapse $\rightarrow$ gas burning \\
\hline 13 & Mechanical friction $\rightarrow$ spark $\rightarrow$ gas explosion \\
\hline 14 & Air blower failure $\rightarrow$ inadequate ventilation $\rightarrow$ gas concentration exceeding limit $\rightarrow$ gas explosion \\
\hline 15 & Electric spark $\rightarrow$ gas burning $\rightarrow$ fire $\rightarrow$ gas explosion \\
\hline 16 & Management negligence $\rightarrow$ violation operation $\rightarrow$ suffocation \\
\hline 17 & Roof collapse $\rightarrow$ mechanical friction $\rightarrow$ spark $\rightarrow$ gas explosion \\
\hline 18 & Inadequate ventilation $\rightarrow$ gas concentration exceeding limit $\rightarrow$ gas explosion \\
\hline 19 & Unreasonable technique scheme $\rightarrow$ geostress concentration $\rightarrow$ coal and gas outburst $\rightarrow$ suffocation \\
\hline 20 & $\begin{array}{l}\text { Imperfect regulation } \rightarrow \text { management negligence } \rightarrow \text { unreasonable blasting } \rightarrow \text { coal and gas outburst } \rightarrow \\
\text { suffocation }\end{array}$ \\
\hline 21 & $\begin{array}{l}\text { Management negligence } \rightarrow \text { poor maintenance } \rightarrow \text { electrical device failure } \rightarrow \text { inadequate ventilation } \rightarrow \text { gas } \\
\text { concentration exceeding limit } \rightarrow \text { gas explosion }\end{array}$ \\
\hline 22 & Unreasonable blasting $\rightarrow$ coal dust explosion $\rightarrow$ carbon monoxide poisoning \\
\hline 23 & Imperfect regulation $\rightarrow$ management negligence $\rightarrow$ electric spark $\rightarrow$ coal dust explosion \\
\hline 24 & Management negligence $\rightarrow$ ruptured steel rope $\rightarrow$ mechanical friction $\rightarrow$ spark $\rightarrow$ gas explosion \\
\hline 25 & Ruptured steel rope $\rightarrow$ mechanical friction $\rightarrow$ spark $\rightarrow$ coal dust explosion \\
\hline 26 & Unreasonable blasting $\rightarrow$ roof collapse $\rightarrow$ ventilation failure $\rightarrow$ coal dust explosion $\rightarrow$ struck-by \\
\hline 27 & Broken steel rope $\rightarrow$ sliding train $\rightarrow$ collision $\rightarrow$ spark $\rightarrow$ coal dust explosion \\
\hline 28 & Violation operation $\rightarrow$ unreasonable blasting $\rightarrow$ naked light $\rightarrow$ gas burning $\rightarrow$ coal dust explosion \\
\hline 29 & Electric spark $\rightarrow$ naked light $\rightarrow$ gas burning $\rightarrow$ fire $\rightarrow$ carbon monoxide poisoning \\
\hline 30 & Electrical failures $\rightarrow$ naked light $\rightarrow$ fire $\rightarrow$ roof collapse \\
\hline 31 & Cable short circuit $\rightarrow$ electric spark $\rightarrow$ fire $\rightarrow$ carbon monoxide poisoning \\
\hline 32 & Electrical failures $\rightarrow$ cable short circuit $\rightarrow$ electric spark $\rightarrow$ fire $\rightarrow$ carbon monoxide poisoning \\
\hline 33 & Violation operation $\rightarrow$ pressure fan failure $\rightarrow$ over-temperature $\rightarrow$ spark $\rightarrow$ fire $\rightarrow$ carbon monoxide poisoning \\
\hline 34 & Inadequate training $\rightarrow$ violation weld $\rightarrow$ naked light $\rightarrow$ fire $\rightarrow$ carbon monoxide poisoning \\
\hline 35 & Management negligence $\rightarrow$ electric spark $\rightarrow$ naked light $\rightarrow$ fire $\rightarrow$ roof collapse \\
\hline 36 & Management negligence $\rightarrow$ conveyor failure $\rightarrow$ over-temperature $\rightarrow$ naked light $\rightarrow$ fire $\rightarrow$ suffocation \\
\hline 37 & $\begin{array}{l}\text { Management negligence } \rightarrow \text { electrical failures } \rightarrow \text { air blower failure } \rightarrow \text { gas concentration exceeding limit } \rightarrow \\
\text { carbon monoxide poisoning }\end{array}$ \\
\hline 38 & Inadequate training $\rightarrow$ unreasonable blasting $\rightarrow$ penetration into goaf $\rightarrow$ water leaking \\
\hline 39 & Violation operation $\rightarrow$ penetration into goaf $\rightarrow$ water leaking \\
\hline 40 & Management negligence $\rightarrow$ unreasonable blasting $\rightarrow$ penetration into goaf $\rightarrow$ water leaking \\
\hline \multirow{2}{*}{41} & Unreasonable blasting $\rightarrow$ gas concentration exceeding limit \\
\hline & Unreasonable blasting $\rightarrow$ naked light $\rightarrow$ gas burning $\rightarrow$ gas explosion \\
\hline 42 & Inadequate geological prospecting $\rightarrow$ unreasonable blasting $\rightarrow$ penetration into goaf $\rightarrow$ water leaking \\
\hline 43 & Inadequate geological prospecting $\rightarrow$ penetration into goaf $\rightarrow$ water leaking \\
\hline
\end{tabular}


TABle 3: Continued.

\begin{tabular}{|c|c|}
\hline Number & Accident chains \\
\hline 44 & Penetration into goaf $\rightarrow$ water leaking \\
\hline 45 & Management negligence $\rightarrow$ violation operation $\rightarrow$ water leaking \\
\hline 46 & Management negligence $\rightarrow$ stagnant water $\rightarrow$ suffocation \\
\hline 47 & Management negligence $\rightarrow$ violation operation $\rightarrow$ penetration into goaf $\rightarrow$ water leaking \\
\hline 48 & Inadequate training $\rightarrow$ standing on conveyor belt $\rightarrow$ fall $\rightarrow$ mechanical injury \\
\hline 49 & Management negligence $\rightarrow$ ruptured steel rope $\rightarrow$ falling of cage \\
\hline 50 & Broken steel rope $\rightarrow$ sliding train $\rightarrow$ train derailment $\rightarrow$ collision \\
\hline 51 & Inadequate training $\rightarrow$ broken steel rope $\rightarrow$ mechanical injury \\
\hline 52 & Unscientific design $\rightarrow$ faulty track $\rightarrow$ train derailment $\rightarrow$ collision \\
\hline 53 & Train overload $\rightarrow$ brake failure $\rightarrow$ sliding train $\rightarrow$ train derailment $\rightarrow$ collision \\
\hline 54 & Management negligence $\rightarrow$ poor maintenance $\rightarrow$ mechanical injury \\
\hline 55 & Violation operation $\rightarrow$ electrical failures $\rightarrow$ electric shock \\
\hline 56 & Management negligence $\rightarrow$ violation operation $\rightarrow$ electric shock \\
\hline 57 & Cable short circuit $\rightarrow$ electric shock \\
\hline 58 & Management negligence $\rightarrow$ inadequate training $\rightarrow$ fall $\rightarrow$ mechanical injury \\
\hline 59 & Stray current $\rightarrow$ gas explosion \\
\hline 60 & Management negligence $\rightarrow$ electric leakage $\rightarrow$ unreasonable blasting \\
\hline 61 & Unscientific design $\rightarrow$ poor tunnel support $\rightarrow$ roof collapse \\
\hline 62 & Float coal $\rightarrow$ tunnel support failure $\rightarrow$ roof collapse \\
\hline 63 & Train derailment $\rightarrow$ collision $\rightarrow$ tunnel support failure $\rightarrow$ roof collapse \\
\hline 64 & Optional withdrawal of pillar $\rightarrow$ roof collapse $\rightarrow$ struck-by \\
\hline 65 & Inadequate geological prospecting $\rightarrow$ neglect of geostress concentration $\rightarrow$ roof collapse \\
\hline 66 & Neglect of geostress concentration $\rightarrow$ roof separation $\rightarrow$ roof collapse \\
\hline 67 & Poor tunnel support $\rightarrow$ flying rock $\rightarrow$ struck-by \\
\hline 68 & Unreasonable blasting $\rightarrow$ tunnel support failure $\rightarrow$ roof collapse \\
\hline 69 & Unscientific design $\rightarrow$ poor tunnel support $\rightarrow$ roof collapse $\rightarrow$ flying rock \\
\hline 70 & Violation operation $\rightarrow$ roof collapse \\
\hline 71 & Unreasonable technique scheme $\rightarrow$ tunnel support failure $\rightarrow$ roof separation $\rightarrow$ roof collapse $\rightarrow$ struck-by \\
\hline 72 & Unreasonable technique scheme $\rightarrow$ unreasonable blasting $\rightarrow$ roof separation $\rightarrow$ roof collapse \\
\hline 73 & Poor tunnel support $\rightarrow$ roof collapse $\rightarrow$ flying rock \\
\hline 74 & Weakness of safe consciousness $\rightarrow$ standing on conveyor belt $\rightarrow$ fall $\rightarrow$ mechanical injury \\
\hline 75 & Roof collapse $\rightarrow$ penetration into goaf $\rightarrow$ carbon monoxide poisoning \\
\hline \multirow{2}{*}{76} & Air blower failure $\rightarrow$ inadequate ventilation $\rightarrow$ gas concentration exceeding limit \\
\hline & Electrical failures $\rightarrow$ cable short circuit $\rightarrow$ electric spark $\rightarrow$ fire \\
\hline 77 & Inadequate geological prospecting $\rightarrow$ neglect of geostress concentration $\rightarrow$ water leaking \\
\hline 78 & Weakness of safe consciousness $\rightarrow$ unreasonable blasting $\rightarrow$ water leaking \\
\hline 79 & Management negligence $\rightarrow$ spontaneous combustion of dynamite $\rightarrow$ dynamite explosion $\rightarrow$ roof collapse \\
\hline 80 & Transformer overload $\rightarrow$ cable short circuit $\rightarrow$ electric spark $\rightarrow$ fire \\
\hline 81 & Sudden torrential rain storm $\rightarrow$ water leaking \\
\hline 82 & Spontaneous combustion of coal seam $\rightarrow$ fire \\
\hline 83 & Unreasonable blasting $\rightarrow$ naked light $\rightarrow$ fire \\
\hline 84 & Violation operation $\rightarrow$ electric shock \\
\hline 85 & Inadequate training $\rightarrow$ violation operation $\rightarrow$ mechanical injury \\
\hline 86 & Unreasonable blasting $\rightarrow$ coal and gas outburst $\rightarrow$ gas explosion \\
\hline 87 & Drilling blasting hole $\rightarrow$ spark $\rightarrow$ gas explosion \\
\hline
\end{tabular}


TABle 3: Continued.

\begin{tabular}{|c|c|}
\hline Number & Accident chains \\
\hline 88 & Violation blasting $\rightarrow$ naked light $\rightarrow$ gas explosion $\rightarrow$ struck-by \\
\hline 89 & Miner's lamp failure $\rightarrow$ electric spark $\rightarrow$ gas explosion \\
\hline \multirow{2}{*}{90} & Unscientific design $\rightarrow$ inadequate ventilation $\rightarrow$ gas concentration exceeding limit \\
\hline & Cable insulation failure $\rightarrow$ cable short circuit $\rightarrow$ electric spark $\rightarrow$ gas explosion \\
\hline 91 & Smoking $\rightarrow$ naked light $\rightarrow$ gas explosion \\
\hline \multirow{2}{*}{92} & Inadequate ventilation $\rightarrow$ gas concentration exceeding limit \\
\hline & Illegal disassembly of miner's miner's lamp $\rightarrow$ electric spark $\rightarrow$ gas explosion \\
\hline \multirow{2}{*}{93} & Defective geological condition $\rightarrow$ coal and gas outburst \\
\hline & Electric locomotive failure $\rightarrow$ electric spark $\rightarrow$ gas explosion \\
\hline 94 & Gas monitoring system failure $\rightarrow$ gas concentration exceeding limit $\rightarrow$ gas explosion \\
\hline 95 & Lack of dedusting device $\rightarrow$ coal dust concentration exceeding limit $\rightarrow$ coal dust explosion \\
\hline 96 & Violation blasting $\rightarrow$ collapse of coal bunker $\rightarrow$ coal dust concentration exceeding limit $\rightarrow$ coal dust explosion \\
\hline 97 & Violation weld $\rightarrow$ conveyor belt burning $\rightarrow$ fire $\rightarrow$ suffocation \\
\hline 98 & Sudden torrential rain storm $\rightarrow$ power cut $\rightarrow$ water pump failure $\rightarrow$ mine flooding \\
\hline \multirow{2}{*}{99} & Power cut $\rightarrow$ ventilation failure $\rightarrow$ gas concentration exceeding limit \\
\hline & Violation blasting $\rightarrow$ naked light $\rightarrow$ gas explosion \\
\hline 100 & Violation blasting $\rightarrow$ poisonous gas leakage $\rightarrow$ poisoning \\
\hline 101 & Severe vibration in coal cutting $\rightarrow$ coal and gas outburst \\
\hline 102 & Explosion of electric switch $\rightarrow$ spark $\rightarrow$ gas explosion \\
\hline \multirow{2}{*}{103} & Roof collapse $\rightarrow$ air blower failure $\rightarrow$ inadequate ventilation $\rightarrow$ gas concentration exceeding limit \\
\hline & Cable short circuit $\rightarrow$ electric spark $\rightarrow$ fire \\
\hline 104 & Metal crash $\rightarrow$ spark $\rightarrow$ gas explosion \\
\hline 105 & Illegal restart $\rightarrow$ electric spark $\rightarrow$ gas explosion \\
\hline \multirow{2}{*}{106} & Optional close of ventilation $\rightarrow$ gas concentration exceeding limit \\
\hline & Illegal disassembly of miner's miner's lamp $\rightarrow$ electric spark $\rightarrow$ gas explosion \\
\hline 107 & Severe vibration in anchor construction $\rightarrow$ coal and gas outburst \\
\hline 108 & Broken steel rope $\rightarrow$ sliding train $\rightarrow$ cable short circuit $\rightarrow$ electric spark $\rightarrow$ coal dust explosion \\
\hline 109 & Mechanical friction $\rightarrow$ spark $\rightarrow$ coal dust explosion \\
\hline 110 & Management negligence $\rightarrow$ electric leakage $\rightarrow$ ignition of cable $\rightarrow$ fire $\rightarrow$ poisonous gas leakage $\rightarrow$ poisoning \\
\hline 111 & Conveyor over-temperature $\rightarrow$ ignition of engine oil $\rightarrow$ spark $\rightarrow$ fire \\
\hline 112 & Violation operation $\rightarrow$ water leaking \\
\hline 113 & Wrong geologic survey $\rightarrow$ wrong holing-through $\rightarrow$ water leaking \\
\hline 114 & Delay of support $\rightarrow$ roof separation $\rightarrow$ roof collapse \\
\hline 115 & Pressure fan failure $\rightarrow$ ignition of engine oil $\rightarrow$ spark $\rightarrow$ fire \\
\hline 116 & Unstable pillar $\rightarrow$ roof separation $\rightarrow$ roof collapse \\
\hline 117 & Winch brake failure $\rightarrow$ falling object $\rightarrow$ struck-by $\rightarrow$ mechanical injury \\
\hline 118 & Trip $\rightarrow$ fall $\rightarrow$ struck-by \\
\hline 119 & Trip $\rightarrow$ mechanical injury \\
\hline 120 & Collapse of support structure $\rightarrow$ roof collapse $\rightarrow$ struck-by \\
\hline 121 & Drinking $\rightarrow$ fall $\rightarrow$ mechanical injury \\
\hline 122 & Violation operation $\rightarrow$ steel rope bouncing $\rightarrow$ mechanical injury \\
\hline \multirow{2}{*}{123} & Management negligence $\rightarrow$ no warning sign \\
\hline & Flying rock $\rightarrow$ struck-by \\
\hline 124 & Management negligence $\rightarrow$ no warning sign $\rightarrow$ entering danger zone $\rightarrow$ suffocation \\
\hline 125 & Entering danger zone $\rightarrow$ flying rock $\rightarrow$ struck-by \\
\hline 126 & Unreasonable dismantling of elevator $\rightarrow$ falling object $\rightarrow$ struck-by \\
\hline
\end{tabular}


TABLE 4: Risk number and type.

\begin{tabular}{|c|c|c|}
\hline Number & Risk & Attribute \\
\hline 1 & Air blower failure & Machine \\
\hline 2 & Asphyxiation & Environment \\
\hline 3 & Delay of support & Management \\
\hline 4 & Brake failure & Machine \\
\hline 5 & Cable insulation failure & Machine \\
\hline 6 & Cable short circuit & Machine \\
\hline 7 & Falling of cage & Machine \\
\hline 8 & Carbon monoxide poisoning & Environment \\
\hline 9 & Optional close of ventilation & Management \\
\hline 10 & Coal and gas outburst & Environment \\
\hline 11 & Collapse of coal bunker & Environment \\
\hline 12 & Coal dust concentration exceeding limit & Environment \\
\hline 13 & Coal dust explosion & Environment \\
\hline 14 & Collapse of support structure & Technology \\
\hline 15 & Collision & Machine \\
\hline 16 & Conveyor belt burning & Machine \\
\hline 17 & Conveyor failure & Machine \\
\hline 18 & Conveyor over-temperature & Machine \\
\hline 19 & Defective geological condition & Environment \\
\hline 20 & Drilling blasting hole & Technology \\
\hline 21 & Drinking & Person \\
\hline 22 & Dynamite explosion & Management \\
\hline 23 & Electric leakage & Machine \\
\hline 24 & Electric locomotive failure & Machine \\
\hline 25 & Electric shock & Machine \\
\hline 26 & Electric spark & Machine \\
\hline 27 & Explosion of electric switch & Machine \\
\hline 28 & Electrical failure & Machine \\
\hline 29 & Electrified device failure & Machine \\
\hline 30 & Entering danger zone & Person \\
\hline 31 & Fall & Person \\
\hline 32 & Falling object & Environment \\
\hline 33 & Faulty track & Machine \\
\hline 34 & Fire & Environment \\
\hline 35 & Float coal & Environment \\
\hline 36 & Flying rock & Environment \\
\hline 37 & Gas burning & Environment \\
\hline 38 & Gas concentration exceeding limit & Environment \\
\hline 39 & Gas explosion & Environment \\
\hline 40 & Gas monitoring system failure & Machine \\
\hline 41 & Geostress concentration & Environment \\
\hline 42 & Ignition of cable & Machine \\
\hline 43 & Ignition of engine oil & Machine \\
\hline 44 & Illegal disassembly of miner's miner's lamp & Person \\
\hline 45 & Illegal restart & Person \\
\hline 46 & Imperfect regulation & Management \\
\hline
\end{tabular}

TABle 4: Continued.

\begin{tabular}{|c|c|c|}
\hline Number & Risk & Attribute \\
\hline 47 & Inadequate geological prospecting & Management \\
\hline 48 & Inadequate training & Management \\
\hline 49 & Inadequate ventilation & Management \\
\hline 50 & Lack of dedusting device & Management \\
\hline 51 & Management negligence & Management \\
\hline 52 & Mechanical friction & Machine \\
\hline 53 & Mechanical injury & Machine \\
\hline 54 & Metal crash & Machine \\
\hline 55 & Mine flooding & Environment \\
\hline 56 & Miner's lamp failure & Machine \\
\hline 57 & Naked light & Machine \\
\hline 58 & Neglect of geostress concentration & Management \\
\hline 59 & No warning sign & Management \\
\hline 60 & Over-temperature & Environment \\
\hline 61 & Penetration into goaf & Technology \\
\hline 62 & Poisoning & Environment \\
\hline 63 & Poisonous gas leakage & Environment \\
\hline 64 & Poor maintenance & Management \\
\hline 65 & Poor tunnel support & Technology \\
\hline 66 & Power cut & Machine \\
\hline 67 & Pressure fan failure & Machine \\
\hline 68 & Roof collapse & Environment \\
\hline 69 & Roof separation & Environment \\
\hline 70 & Ruptured steel rope & Machine \\
\hline 71 & Severe vibration in anchor construction & Technology \\
\hline 72 & Severe vibration in coal cutting & Technology \\
\hline 73 & Sliding train & Machine \\
\hline 74 & Smoking & Person \\
\hline 75 & Spark & Machine \\
\hline 76 & Spontaneous combustion of coal seam & Environment \\
\hline 77 & Spontaneous combustion of Dynamite & Management \\
\hline 78 & Stagnant water & Environment \\
\hline 79 & Standing on conveyor belt & Person \\
\hline 80 & Steel rope bouncing & Machine \\
\hline 81 & Broken steel rope & Machine \\
\hline 82 & Stray current & Machine \\
\hline 83 & Struck-by & Person \\
\hline 84 & Sudden torrential rain storm & Environment \\
\hline 85 & Train derailment & Machine \\
\hline 86 & Train overload & Machine \\
\hline 87 & Transformer overload & Machine \\
\hline 88 & Trip & Person \\
\hline 89 & Tunnel support failure & Technology \\
\hline 90 & Unreasonable blasting & Technology \\
\hline 91 & Unreasonable dismantling of elevator & Technology \\
\hline 92 & Unreasonable technique scheme & Technology \\
\hline
\end{tabular}


TABLE 4: Continued.

\begin{tabular}{lcc}
\hline Number & Risk & Attribute \\
\hline 93 & Unscientific design & Technology \\
94 & Unstable pillar & Technology \\
95 & Ventilation failure & Machine \\
96 & Violation blasting & Management \\
97 & Violation operation & Management \\
98 & Violation weld & Management \\
99 & Water leaking & Environment \\
100 & Water pump failure & Machine \\
101 & Weakness of safe consciousness & Person \\
102 & Winch brake failure & Machine \\
103 & Optional withdrawal of pillar & Management \\
104 & Wrong geologic survey & Technology \\
105 & Wrong holing-through & Technology \\
\hline
\end{tabular}

an overall perspective. It specifically refers to the proportion of actual edges to potential edges in a network. Consisting of 105 vertexes, the maximum number of edges in CMRN should be $105 * 104=10920$. Since the actual edges in CMRN is 194, thus the network density of CMRN is $194 / 10920=0.178$. In general, the more the vertexes, the smaller the network density. Low density means that CMRN is a relatively sparse network. Moreover, the vertex in CMRN is less connected with all others. That is to say, the degree of a vertex in CMRN directly affected by others is relatively low.

4.1.3. Average Path Length. The transmission efficiency of information or energy is significantly correlated with the average path length. A shorter average path length means higher efficiency. The average path length can be defined as the average number of steps between all possible pairs of vertexes in a network. The value of the average path length in CMRN is 3.0841, indicating that a risk can transmit to another only in three steps on average. For example, cable short circuit (vertex 6) and carbon monoxide poisoning (vertex 8) refer to two correlative risks, which can be connected by electric spark (vertex 26) and fire (vertex 34) in three steps, as shown in accident 31 in Table 3.

4.1.4. Degree. The degree of a vertex is defined as the number of edges connected to the vertex. In a directed network, the degree can be either in-degree (number of incoming edges) or out-degree (number of outgoing edges), with the total degree being the sum of the two. Since there are 105 vertexes in CMRN, it is impossible to show all the vertex degree in a radar graph. Consequently, 30 vertexes with the highest degree are selected as the example to display vertex degree. The values of the in-degree, out-degree, and total degree of these 30 vertexes are presented in Figure 6. Roof collapse (vertex 68) has the highest degree of 17, with an in-degree 10 and out-degree 7. This indicates that the roof collapse is in a relatively central position and plays a critical role in the accident chain. Its in-degree is also the highest in the network, implying that it refers to the biggest "risk recipient" in CMRN and many risks such as poor tunnel support can lead to roof collapse. Multiple paths make it difficult to control for roof collapse, compared to other vertexes with low in-degree. The second is unreasonable blasting (vertex 90) and the third is the management negligence (vertex 51). The in-degree and out-degree of unreasonable blasting are 7 and 9, respectively. It means that 7 risks could give rise to unreasonable blasting, and meanwhile, unreasonable blasting might cause 9 risks in production. Additionally, the management negligence (vertex 51) has the highest outdegree, demonstrating that management negligence is the most serious risk source. If there is something wrong in safety management, many risks might be triggered at any time, such as gas concentration exceeding limit. Controlling these key vertexes can positively influence the safety of coal mine, which is also referential in resource distribution under the condition of limited security resource. Besides, it would greatly help disrupt the connectivity among risks to prevent risks from spreading and propagating in CMRN.

4.1.5. Betweenness. Betweenness is used to describe the extent to which a vertex plays an intermediary role in the interaction between all possible pairs of vertexes in a network [38]. Two types of betweenness, vertex betweenness and edge betweenness, are used extensively in the network analysis [39, 40]. According to the research object, only vertex betweenness is utilized in this study. High betweenness indicates greater importance in the whole network. The vertex betweenness in the CMRN ranges from 0 to 0.059852 , as shown in Table 5. Only 47 vertexes are invisible because their vertex betweenness is zero, which indicates that they do not play the role of intermediary among interactions between other vertexes. The roof collapse (vertex 68) has the highest value of vertex betweenness, meaning that the maximum number of the shortest paths passes through roof collapse (vertex 68). It is a key link in the process of risk spread. The stagnant water (vertex 78) has the lowest value of vertex betweenness, meaning that the minimum number of the shortest paths passes through stagnant water (vertex 78). It is not a key link in the process of risk spread. According to the value of betweenness, the impact of roof collapse (vertex 68) is much larger than stagnant water (vertex 78) in the process of risk spread. Furthermore, fire (vertex 34) and spark (vertex 75) are 0.048486 and 0.020668 , respectively. The cumulative vertex betweenness of the five highest vertex betweenness is equal to 0.542701 , which indicates that about $55 \%$ shortest paths pass through these five vertexes. These vertexes should be focused in the safety management. It seems that effectively controlling these few key vertexes can slow down the risk diffusion and decrease the chain reaction in CMRN.

4.1.6. Clustering Coefficient. The clustering coefficient is used to describe which vertexes in a network tend to cluster together from a local perspective [41]. The clustering coefficient of a vertex is defined as the probability of two randomly selected neighbors of the vertex being connected. It can be found that 33 vertexes get the missing value of 999999998 because the degrees of these vertexes are equal to 1 , and 34 vertexes have the value of 0 . The clustering coefficients of 


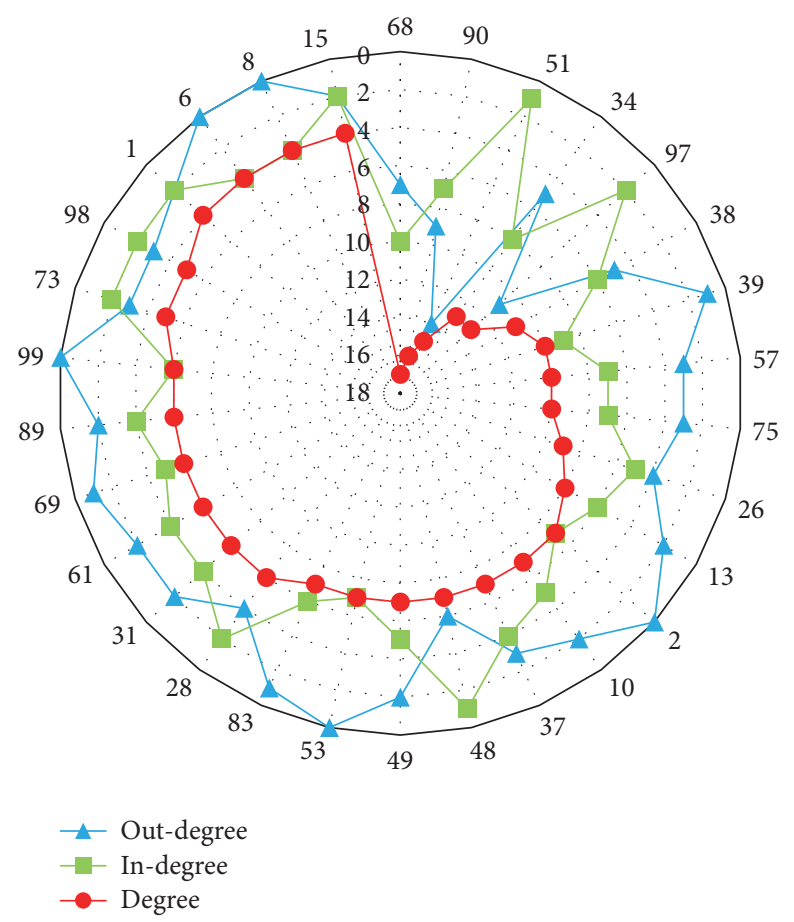

FIGURE 6: In-degree, out-degree, and total degree values.

other 38 vertexes are presented from high to low in Table 6. The clustering coefficient of vertex in CMRN ranges from 0 to 0.5 . The vertexes with the highest clustering coefficient are vertex 25 and vertex 80 . The network clustering coefficient can be defined as the average value of all vertexes in the network, and it is 0.0623 in CMRN which is larger than a random network with the same network scale. The large clustering coefficient denotes that CMRN has a high degree of cliquishness.

4.2. Network Property. With the development of network theory, it can be found that small-world property and scalefree property are the most obvious distinction between real network and random network. To obtain greater insight into the nature of CMRN, this section explores these two properties.

4.2.1. Small-World Property. A small-world network is a special kind of graph, in which most vertexes can be reached from every other vertex by a short path. In general, smallworld network is associated with the possession of relatively high value of clustering coefficient and small average path length $[42,43]$. For comparison, three random networks with 105 vertexes and 194 edges are created by Pajek, which are the same scale as CMRN. The clustering coefficient and average path length of CMRN and random networks are presented in Table 7. Obviously, CMRN is a relatively small-world network according to its clustering coefficient and average path length, indicating that the risk propagation in CMRN is much faster than a random network. To avoid a worse consequence under the condition of an occurred accident, controlling the catenation among accidents is of great significance.
4.2.2. Scale-Free Property. A scale-free network is a network whose degree distribution satisfies power-law decay. In such network, numerous vertexes are poorly connected and relatively few vertexes are linked to many other vertexes [44]. Due to rare vertexes with high degree, analyzing statistic data in the tail of the degree distribution is meaningless. The degree distribution $P(k)$ is defined as the proportion of vertexes with degree $k$, while the cumulative $P(k)$ is defined as the proportion of vertexes equaling to or greater than $k$ [45]. In practice, the cumulative $P(k)$ is preferred in statistical analysis using double logarithmic coordinate system, with the purpose of reducing statistical errors caused by finite network size [46]. The cumulative $P(k)$ of $C M R N$ is depicted in Figure 7 with approximate fit $P(k)=2.1217 \times k^{-1.545}$, which basically follows the power-law. This indicates that the CMRN has scale-free property according to complex network theory. The property means that CMRN is robust to random risks to some extent. The vertex with degree equaling to or less than 4 accounts for $75 \%$, and the influence of these vertexes on the network is relatively small. However, CMRN is vulnerable to simultaneous attacks aiming at vertexes with high degree. In other words, only targeted actions can greatly prevent the cascading effects in CMRN.

4.3. Measuring the Effect of Risk Control. The analysis on effect of risk control is conductive to providing recommendations and proposal for safety management in coal mine. To measure the effect of risk control, an assumption is made. Namely, a risk would be supposed not to occur if it is completely controlled in coal mine production. Furthermore, if a risk will not happen, it can be deleted from CMRN. Then, the effect of risk control can be measured by network global 
TABLE 5: Betweenness in CMRN.

\begin{tabular}{|c|c|}
\hline Vertex & Betweenness \\
\hline 68 & 0.059852 \\
\hline 34 & 0.048486 \\
\hline 75 & 0.020668 \\
\hline 38 & 0.016303 \\
\hline 15 & 0.012481 \\
\hline 89 & 0.010105 \\
\hline 90 & 0.009873 \\
\hline 57 & 0.009704 \\
\hline 26 & 0.008742 \\
\hline 1 & 0.008044 \\
\hline 73 & 0.007534 \\
\hline 37 & 0.007143 \\
\hline 83 & 0.007001 \\
\hline 61 & 0.006588 \\
\hline 95 & 0.006566 \\
\hline 63 & 0.006068 \\
\hline 52 & 0.005903 \\
\hline 39 & 0.005813 \\
\hline 51 & 0.005134 \\
\hline 13 & 0.005128 \\
\hline 49 & 0.004844 \\
\hline 69 & 0.003734 \\
\hline 60 & 0.003423 \\
\hline 97 & 0.002563 \\
\hline 43 & 0.002558 \\
\hline 85 & 0.002474 \\
\hline 4 & 0.002427 \\
\hline 66 & 0.001960 \\
\hline 10 & 0.001875 \\
\hline 22 & 0.001867 \\
\hline 29 & 0.001774 \\
\hline 48 & 0.001147 \\
\hline 65 & 0.000996 \\
\hline 81 & 0.000937 \\
\hline 28 & 0.000850 \\
\hline 12 & 0.000794 \\
\hline 16 & 0.000770 \\
\hline 98 & 0.000745 \\
\hline 31 & 0.000742 \\
\hline 67 & 0.000660 \\
\hline 36 & 0.000560 \\
\hline 58 & 0.000490 \\
\hline 70 & 0.000467 \\
\hline 30 & 0.000436 \\
\hline 33 & 0.000420 \\
\hline 23 & 0.000373 \\
\hline 32 & 0.000373 \\
\hline 17 & 0.000280 \\
\hline 64 & 0.000280 \\
\hline 59 & 0.000249 \\
\hline 79 & 0.000218 \\
\hline 77 & 0.000187 \\
\hline 100 & 0.000187 \\
\hline 42 & 0.000179 \\
\hline 11 & 0.000140 \\
\hline 41 & 0.000101 \\
\hline
\end{tabular}

TABle 5: Continued.

\begin{tabular}{lc}
\hline Vertex & Betweenness \\
\hline 105 & 0.000093 \\
78 & 0.000062 \\
$\vdots$ & 0 \\
\hline \multicolumn{2}{c}{ TABLE 6: Clustering coefficient in CMRN. }
\end{tabular}

\begin{tabular}{|c|c|}
\hline Vertex & Clustering coefficient \\
\hline 25 & 0.5000 \\
\hline 80 & 0.5000 \\
\hline 88 & 0.5000 \\
\hline 61 & 0.2000 \\
\hline 36 & 0.1667 \\
\hline 47 & 0.1667 \\
\hline 65 & 0.1667 \\
\hline 79 & 0.1667 \\
\hline 85 & 0.1667 \\
\hline 92 & 0.1667 \\
\hline 37 & 0.1429 \\
\hline 69 & 0.1333 \\
\hline 89 & 0.1333 \\
\hline 48 & 0.1190 \\
\hline 31 & 0.1000 \\
\hline 39 & 0.1000 \\
\hline 98 & 0.1000 \\
\hline 99 & 0.1000 \\
\hline 49 & 0.0952 \\
\hline 1 & 0.0833 \\
\hline 8 & 0.0833 \\
\hline 15 & 0.0833 \\
\hline 23 & 0.0833 \\
\hline 58 & 0.0833 \\
\hline 97 & 0.0833 \\
\hline 2 & 0.0714 \\
\hline 53 & 0.0714 \\
\hline 34 & 0.0705 \\
\hline 28 & 0.0667 \\
\hline 57 & 0.0667 \\
\hline 38 & 0.0636 \\
\hline 90 & 0.0583 \\
\hline 73 & 0.0500 \\
\hline 83 & 0.0476 \\
\hline 26 & 0.0417 \\
\hline 68 & 0.0368 \\
\hline 51 & 0.0333 \\
\hline 75 & 0.0111 \\
\hline$\vdots$ & 0 \\
\hline
\end{tabular}

efficiency. Although many definitions on network global efficiency are currently created and studied, they all have different limitations. The generally accepted measure method is average reciprocal shortest path lengths of networks, in 
TABLE 7: The comparison between CMRN and random networks.

\begin{tabular}{lcc}
\hline Network model & Clustering coefficient & Average path length \\
\hline CMRN & 0.0623 & 3.0841 \\
Random network 1 & 0.0156 & 5.6134 \\
Random network 2 & 0.0189 & 5.8130 \\
Random network 3 & 0.0152 & 5.4532 \\
\hline
\end{tabular}

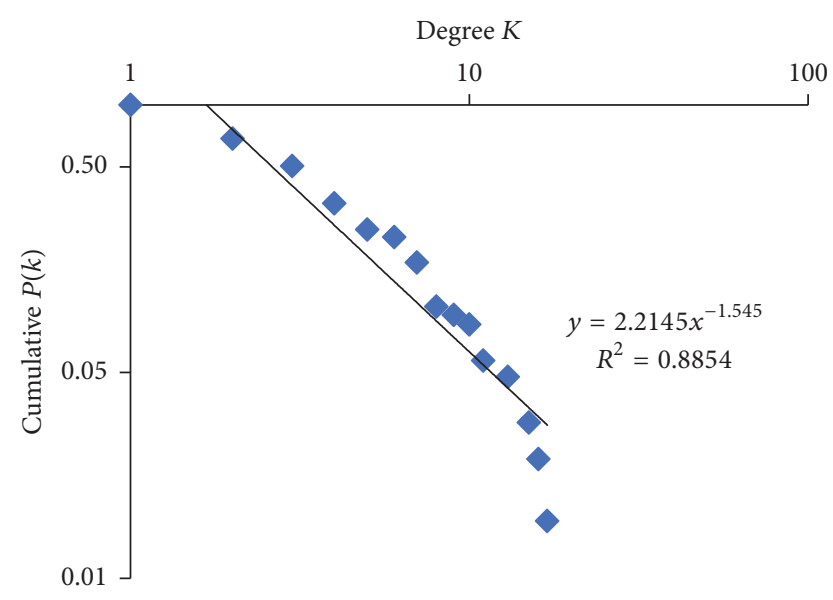

FIgURE 7: Cumulative degree distribution of CMRN.

which network global efficiency of a network $G$ could be calculated by (1) $[47,48]$, where $n$ refers to the number of vertexes and $d_{i j}$ refers to the distance between two vertexes.

$$
E(G)=\frac{1}{n(n-1)} \sum_{\forall i, j, i \neq j} \frac{1}{d_{i j}}
$$

The effect of risk control of every risk in CMRN can be measured according to the degree of network global efficiency declined. For example, if the network global efficiency decreases by 0.1 after deletion of vertex 8 , it means that the effect of risk control of vertex 8 is 0.1 . The better the effect is, the greater the risk will be. The 30 most serious risks in CMRN are identified through calculation, and the risk control effects can be shown in Figure 8. It is observed that roof collapse is the most serious risk and controlling roof collapse could help decrease $32.63 \%$ of network global efficiency of CMRN, followed by fire (25.96\%) and gas concentration exceeding limit $(11.22 \%)$. However, due to the interaction between roof collapse and gas concentration exceeding limit, the effect of controlling "roof collapse" and "gas concentration exceeding limit" is not equal to $32.63 \%$ plus $25.96 \%$, but $44.03 \%$ by calculation. Obviously, measuring the effect of risk control can suggest and designate the directions and key points to further safety management. Anyway, controlling several most serious risks is the most appropriate and most effective approach for preventing accident and further promoting the safety management level in the coal mine production.

\section{Discussion}

Based on the network theory, an analytical framework has been put forward to promote coal mine production safety, which turns out to be feasible and effective. The proposed network modeling method is a powerful and promising tool to analyze risk in various disciplines. It is envisaged that this study can help managerial personnel deeply understand coal mine risk for the sake of developing necessary strategies that can improve safety management in a dynamic operating environment, especially in emergency.

The potential contributions of this study include four aspects. First, it is beneficial to understand the complexity and transitivity of risks in coal mine. The main topology properties and network properties of CMRN are captured and analyzed. Second, it is conductive to enhance the safety performance by controlling original risks and avoiding derivative accidents. Third, this study has the potential benefits in coal mine emergency and relief, which can help managers make decisions in emergency rescue for lightening the casualties and losses. Additionally, network modeling technique is employed in this study, which may offer a promising approach for the analysis of the accident. Also, the application range of network theory will be enlarged.

The main limitation of this research is that the established network model fails to take the vertex weight into consideration. Moreover, the frequency of risks in Table 3 cannot reflect the vertex weight in current study, and it is very difficult to discern and distinguish the different importance for different risks. Therefore, assigning the weight is quite difficult. That may explain why the network model in this study is unweighted. In the future study, more attention should be paid to improve the network model based on more precise understanding of risks in coal mine. Also, how to reduce the risks in coal mine is a significant direction that deserves further research. Meanwhile, a particular failure knowledge database (FKD) would be significant in studying the coal mine accidents, which is the foundation of case based reasoning (CBR) for analyzing hazard and risk.

What is more, several identified risks seem to be general, rather than specific. There are two reasons for this result. First, the risk identification is carried out on the basis of accident data collected from literature and media. If some detailed information is ignored and not recorded during the investigation of these accidents, the unavailable information may affect the accuracy of risk identification. Second, this research is implemented from a holistic perspective. If the identified risks are too specific, finding the common vertex and constructing the coal mine risk network will be difficult. 


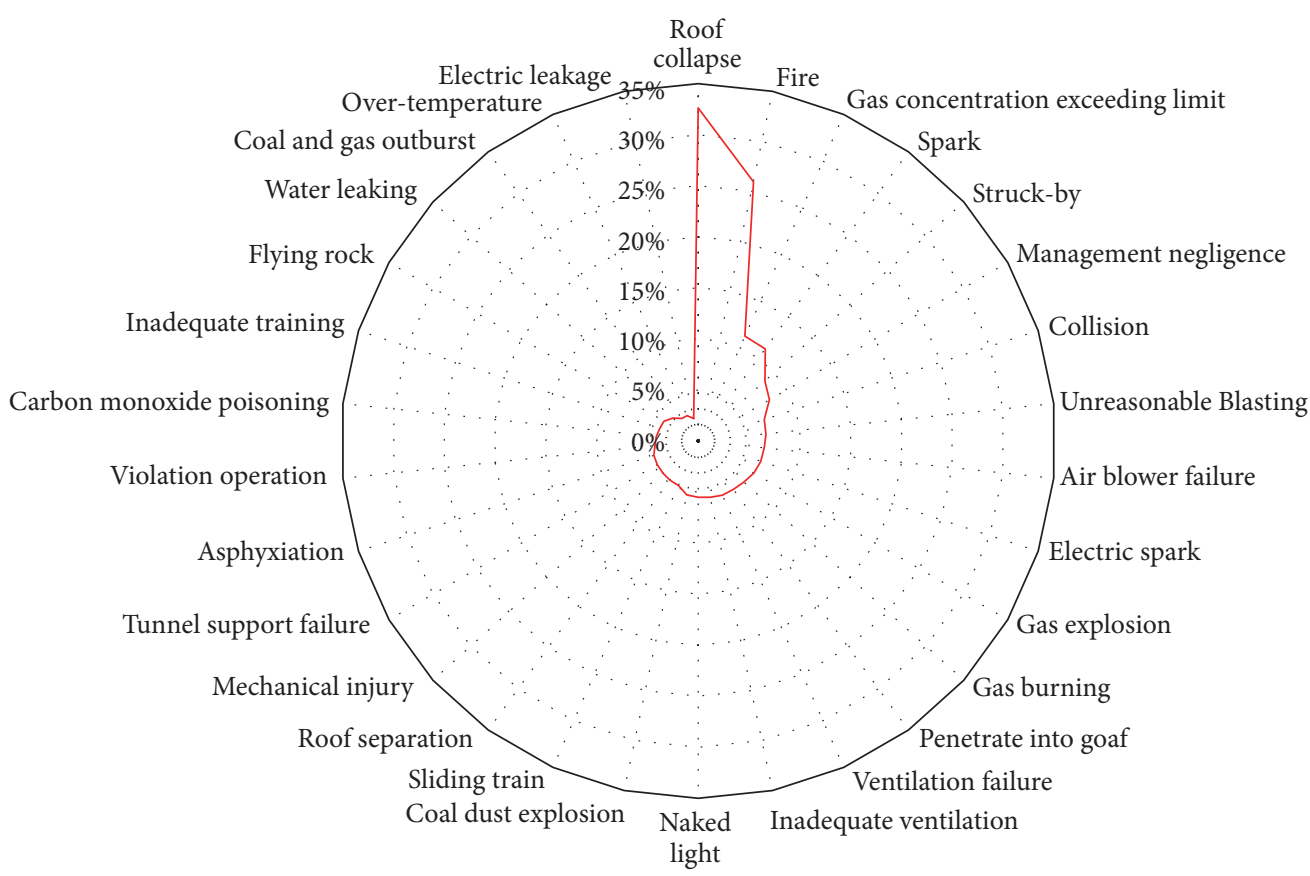

FIGURE 8: The effect of risk control of 30 most serious risks.

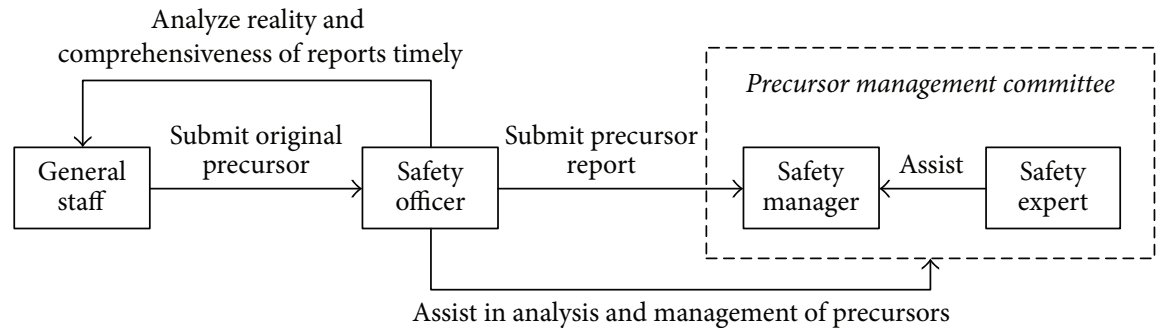

FIGURE 9: The proposed organization structure.

Hence, the similar risks are divided into the same category for the sake of convenience.

Safety researchers assiduously aim to lower the prevalence of accident and raise the safety level. Accident precursor is studied in various industries, and many studies indicate that a series of precursors always occur before the accident $[8,11,49]$. Therefore, lowering precursor frequency is an effective approach to reduce the accident probability [50]. An accident precursor is broadly defined as the "conditions, events, and sequences that precede and lead up to an accident" by the National Academy of Engineering [51]. Even though it is impossible to completely prevent coal mine accident, monitoring and controlling precursory information is a useful and effective approach for safety managers to identify hazards or risks in advance. Also, this can reduce the possibility of accident or alleviate their consequence. Hence, precursor analysis seemingly has huge potential to promote safety management of coal mine production.

According to the results of network analysis, it can be known that some key vertexes play an important role in accident prevention. In practice, precursor can be used to reduce the frequency and probability of these key risks. For example, the precursor of water leaking mainly includes the following: air turns cold; mist appears on the roadway; and coal wall has water seepage. If the coal miner can pay more attention to these precursors, the water leaking will be reduced to a large extent. Therefore, an organization structure should be proposed to manage and control precursors, as depicted in Figure 9. The real executors of precursor management include general staff, safety manager, and safety expert. General staff should report precursor to the safety officer, and then safety officer submits precursor reports to the safety manager. Furthermore, safety expert assists safety manager to analyze risk as well as factor and propose processing measures. The proposed measures or solutions are executed by general staff and safety officer, and meanwhile, the evaluation of them is implemented by safety manager and safety expert. The coal mine enterprise can set up a committee, mainly including safety manager and safety expert, to deal with precursor management.

\section{Conclusion}

The accidents in the coal industry have been widely analyzed to promote safety production. By changing the original 
method of analyzing a single accident, this research aims to develop an innovative approach of fusing various risks that can explore the full complexity of CMRN based on network theory.

The CMRN is constructed by software Pajek based on 135 typical accident chains, which are obtained from 126 typical accidents in coal mine accident database (CMAD). As an unweighted directed network model, CMRN includes 105 vertexes and 194 edges. The network diameter in the CMRN is 7 and the network density of CMRN is 0.178 , which indicates that CMRN refers to a relatively sparse network. The value of the average path length in CMRN is 3.0841, suggesting that a risk can transmit to another only in three steps on average. Roof collapse (vertex 68) has the highest degree of 17 , which indicates that roof collapse plays a critical role in the accident chain. In general, this type of vertex is regarded as a key point. The vertex betweenness in the CMRN ranges from 0 to 0.059852 . Additionally, the roof collapse (vertex 68) has the highest value of vertex betweenness, which means that the maximum number of shortest paths passes through roof collapse (vertex 68). It is a key link in the process of risk spread. Next, fire (vertex 34 ) and spark (vertex 75 ) are 0.048486 and 0.020668 , respectively. About 55\% shortest paths pass through these five highest betweenness vertexes. Effectively controlling roof collapse, fire, spark, gas concentration exceeding limit, and collision could not only increase the network diameter and average path length but also slow down the efficiency of accident propagation and weaken the chain reaction. The vertex clustering coefficient in $\mathrm{CMRN}$ ranges from 0 to 0.5 . Moreover, the clustering coefficient of CMRN is 0.0623 in CMRN, which denotes that CMRN has a high degree of cliquishness. Besides, CMRN is a relatively small-world network according to its clustering coefficient and average path length, demonstrating that the risk propagation in CMRN is much faster than a random network. CMRN also has the scale-free property because cumulative $P(k)$ follows the power-law. The property indicates that CMRN is robust to random risks to some extent. Furthermore, the effect of risk control is calculated precisely. Overall, roof collapse, fire, and gas concentration exceeding limit are not only three most valuable targets in safety management but also the three most dangerous risks in coal mine production.

Precise calculation of these six parameters and effective risk control are beneficial to capture the complexity and nature of coal mine accident and designate the targets for risk control. Also, the results can help promote coal mine safety management in controlling original risks and preventing derivative accidents. In view of the sequential interrelations among accidents in CMRN, this research may also positive influence the early warning of accidents. In practice, the safety managers should focus more on the identified and valuable targets of risk control and put more resources to help promote safety performance in coal mine production.

\section{Conflicts of Interest}

The authors declare that there are no conflicts of interest regarding the publication of this paper.

\section{Acknowledgments}

The authors also gratefully acknowledge those who provided data and suggestions. The research described in this paper is supported by National Natural Science Foundation of China (51323004), the Humanities and Social Sciences Youth Foundation of China's Education Ministry (17YJCZH035), the Fundamental Research Funds for the Central Universities (2017QNB13), and Jiangsu Planned Projects for Postdoctoral Research Funds (1701143C).

\section{References}

[1] S. Niu, "Coal mine safety production situation and management strategy," Management and Engineering, vol. 14, 78 pages, 2014.

[2] P. S. Paul, "Predictors of work injury in underground mines - an application of a logistic regression model," Mining Science and Technology, vol. 19, no. 3, pp. 282-289, 2009.

[3] V. V. Khanzode, J. Maiti, and P. K. Ray, "A methodology for evaluation and monitoring of recurring hazards in underground coal mining," Safety Science, vol. 49, no. 8, pp. 1172-1179, 2011.

[4] A. Nieto, Y. Gao, L. Grayson, and G. Fu, "A comparative study of coal mine safety performance indicators in China and the USA," International Journal of Mining and Mineral Engineering, vol. 5, no. 4, pp. 299-314, 2014.

[5] Q. Liu, X. Meng, M. Hassall, and X. Li, "Accident-causing mechanism in coal mines based on hazards and polarized management," Safety Science, vol. 85, pp. 276-281, 2016.

[6] S. Mahdevari, K. Shahriar, and A. Esfahanipour, "Human health and safety risks management in underground coal mines using fuzzy TOPSIS," Science of the Total Environment, vol. 488-489, no. 1, pp. 85-99, 2014.

[7] H. Chen, Q. Feng, R. Long, and H. Qi, "Focusing on coal miners' occupational disease issues: A comparative analysis between China and the United States," Safety Science, vol. 51, no. 1, pp. 217-222, 2013.

[8] Z. Zhou, Q. Li, and W. Wu, "Developing a versatile subway construction incident database for safety management," Journal of Construction Engineering and Management, vol. 138, no. 10, pp. 1169-1180, 2011.

[9] J. Santos-Reyes and A. N. Beard, "A systemic analysis of the Edge Hill railway accident," Accident Analysis \& Prevention, vol. 41, no. 6, pp. 1133-1144, 2009.

[10] J. K. Wachter and P. L. Yorio, "A system of safety management practices and worker engagement for reducing and preventing accidents: an empirical and theoretical investigation," Accident Analysis \& Prevention, vol. 68, pp. 117-130, 2014.

[11] A. Al-shanini, A. Ahmad, and F. Khan, "Accident modelling and analysis in process industries," Journal of Loss Prevention in the Process Industries, vol. 32, pp. 319-334, 2014.

[12] Z. Zhou, J. Irizarry, and Q. Li, "Using network theory to explore the complexit of subway construction accident network (SCAN) for promoting safety management," Safety Science, vol. 64, pp. 127-136, 2014.

[13] P. Andrews-Speed, M. Yang, L. Shen, and S. Cao, “The regulation of China's township and village coal mines: A study of complexity and ineffectiveness," Journal of Cleaner Production, vol. 11, no. 2, pp. 185-196, 2003.

[14] H. Chen, H. Qi, R. Long, and M. Zhang, "Research on 10-year tendency of China coal mine accidents and the characteristics of human factors," Safety Science, vol. 50, no. 4, pp. 745-750, 2012. 
[15] H. Chen, Q. Feng, and J. Cao, "Rent-seeking mechanism for safety supervision in the Chinese coal industry based on a tripartite game model," Energy Policy, vol. 72, pp. 140-145, 2014.

[16] N. A. Grazhevska, A. Virchenko, and A. Grazhevska, "The effects of rent-seeking behavior on the efficiency of fiscal policy in Ukraine," Procedia Economics and Finance, vol. 27, pp. 274287, 2015.

[17] T.-H. Kwon, "Rent and rent-seeking in renewable energy support policies: feed-in tariff vs. renewable portfolio standard," Renewable \& Sustainable Energy Reviews, vol. 44, pp. 676-681, 2015.

[18] H. Chen, Q. Feng, D. Zhu, S. Han, and R. Long, "Impact of rent-seeking on productivity in Chinese coal mine safety supervision: a simulation study, Energy Policy, vol. 93, pp. 315329, 2016.

[19] M. Sari, A. S. Selcuk, C. Karpuz, and H. S. B. Duzgun, "Stochastic modeling of accident risks associated with an underground coal mine in Turkey," Safety Science, vol. 47, no. 1, pp. 78-87, 2009.

[20] C. Qing-gui, L. Kai, L. Ye-jiao, S. Qi-hua, and Z. Jian, "Risk management and workers' safety behavior control in coal mine," Safety Science, vol. 50, no. 4, pp. 909-913, 2012.

[21] I. J. Kowalska, "Risk management in the hard coal mining industry: social and environmental aspects of collieries' liquidation," Resources Policy, vol. 41, no. 1, pp. 124-134, 2014.

[22] A. Badri, S. Nadeau, and A. Gbodossou, "A new practical approach to risk management for underground mining project in Quebec," Journal of Loss Prevention in the Process Industries, vol. 26, no. 6, pp. 1145-1158, 2013.

[23] L. Wang, Y. Wang, Q. Cao, X. Li, J. Li, and X. Wu, "A framework for human error risk analysis of coal mine emergency evacuation in China," Journal of Loss Prevention in the Process Industries, vol. 30, no. 1, pp. 113-123, 2014.

[24] Q.-L. Liu and X.-C. Li, "Modeling and evaluation of the safety control capability of coal mine based on system safety," Journal of Cleaner Production, vol. 84, no. 1, pp. 797-802, 2014.

[25] E. Ghasemi, M. Ataei, K. Shahriar, F. Sereshki, S. E. Jalali, and A. Ramazanzadeh, "Assessment of roof fall risk during retreat mining in room and pillar coal mines," International Journal of Rock Mechanics and Mining Sciences, vol. 54, pp. 80-89, 2012.

[26] L. M. Pejic, J. G. Torrent, E. Querol, and K. Lebecki, "A new simple methodology for evaluation of explosion risk in underground coal mines," Journal of Loss Prevention in the Process Industries, vol. 26, no. 6, pp. 1524-1529, 2013.

[27] A. Bahri Najafi, G. R. Saeedi, and M. A. Ebrahimi Farsangi, "Risk analysis and prediction of out-of-seam dilution in longwall mining," International Journal of Rock Mechanics and Mining Sciences, vol. 70, pp. 115-122, 2014.

[28] J. Chen, L. Ma, C. Wang, H. Zhang, and M. Ha, "Comprehensive evaluation model for coal mine safety based on uncertain random variables," Safety Science, vol. 68, pp. 146-152, 2014.

[29] D. V. Petrović, M. Tanasijević, V. Milić, N. Lilić, S. Stojadinović, and I. Svrkota, "Risk assessment model of mining equipment failure based on fuzzy logic," Expert Systems with Applications, vol. 41, no. 18, pp. 8157-8164, 2014.

[30] D. R. Lokhande, V. M. S. R. Murthy, V. Vellanky, and B. K. Singh, "Assessment of pot-hole subsidence risk for Indian coal mines," International Journal of Mining Science and Technology, vol. 25, no. 2, article no. 467, pp. 185-192, 2015.

[31] M. Spada and P. Burgherr, "An aftermath analysis of the 2014 coal mine accident in Soma, Turkey: Use of risk performance indicators based on historical experience," Accident Analysis \& Prevention, vol. 87, pp. 134-140, 2016.

[32] E. Sun, X. Zhang, and Z. Li, “The internet of things (IOT) and cloud computing (CC) based tailings dam monitoring and prealarm system in mines," Safety Science, vol. 50, no. 4, pp. 811-815, 2012.

[33] K. M. Dange and R. T. Patil, "Design of monitoring system for coal mine safety based on MSP430," International Journal of Engineering Science Invention, vol. 2, no. 7, pp. 14-19, 2013.

[34] C. Bo, C. Xin, Z. Zhongyi, Z. Chengwen, and C. Junliang, "Web of things-based remote monitoring system for coal mine safety using wireless sensor network," International Journal of Distributed Sensor Networks, vol. 2014, Article ID 323127, 14 pages, 2014.

[35] Y. Zhang, W. Yang, D. Han, and Y.-I. Kim, "An integrated environment monitoring system for underground coal minesWireless Sensor Network subsystem with multi-parameter monitoring," Sensors, vol. 14, no. 7, pp. 13149-13170, 2014.

[36] J. Xu, H. Gao, J. Wu, and Y. Zhang, "Improved safety management system of coal mine based on iris identification and RFID technique," in Proceedings of the IEEE International Conference on Computer and Communications, ICCC 2015, pp. 260-264, China, October 2015.

[37] W. De Nooy, A. Mrvar, and V. Batagelj, Exploratory Social Network Analysis with Pajek, vol. 27, Cambridge University Press, 2011.

[38] B. W. Wambeke, M. Liu, and S. M. Hsiang, "Using Pajek and centrality analysis to identify a social network of construction trades," Journal of Construction Engineering and Management, vol. 138, no. 10, pp. 1192-1201, 2011.

[39] A. Abbasi, L. Hossain, and L. Leydesdorff, "Betweenness centrality as a driver of preferential attachment in the evolution of research collaboration networks," Journal of Informetrics, vol. 6, no. 3, pp. 403-412, 2012.

[40] A. M. M. González, B. Dalsgaard, and J. M. Olesen, “Centrality measures and the importance of generalist species in pollination networks," Ecological Complexity, vol. 7, no. 1, pp. 36-43, 2010.

[41] Almaas, E., Kulkarni, R. V., \&amp; Stroud, D. (2002). Characterizing the structure of small-world networks. Physical review letters, 88(9), 098101.

[42] S. V. Buldyrev, R. Parshani, G. Paul, H. E. Stanley, and S. Havlin, "Catastrophic cascade of failures in interdependent networks," Nature, vol. 464, no. 7291, pp. 1025-1028, 2010.

[43] D. J. Watts and S. H. Strogatz, "Collective dynamics of 'smallworld' networks," Nature, vol. 393, no. 6684, pp. 440-442, 1998.

[44] A. Barabasi and R. Albert, "Emergence of scaling in random networks," Science, vol. 286, no. 5439, pp. 509-512, 1999.

[45] S. Ghosh, A. Banerjee, N. Sharma et al., "Statistical analysis of the Indian railway network: a complex network approach," Acta Physica Polonica B, vol. 4, no. 2, pp. 123-137, 2011.

[46] M. E. Newman, "Assortative mixing in networks," Physical Review Letters, vol. 89, no. 20, Article ID 208701, 2002.

[47] P. Crucittia, V. Latorab, M. Marchioric, and A. Rapisarda, "Efficiency of scale-free networks: error and attack tolerance," Physica A: Statistical Mechanics and its Applications, vol. 320, pp. 622-642, 2003.

[48] R. Criado, A. García del Amo, B. Hernández-Bermejo, and M. Romance, "New results on computable efficiency and its stability for complex networks," Journal of Computational and Applied Mathematics, vol. 192, no. 1, pp. 59-74, 2006. 
[49] N. Khakzad, F. Khan, and P. Amyotte, "Major accidents (gray swans) likelihood modeling using accident precursors and approximate reasoning," Risk Analysis, vol. 35, no. 7, pp. 13361347, 2015.

[50] M. Kyriakidis, R. Hirsch, and A. Majumdar, "Metro railway safety: an analysis of accident precursors," Safety Science, vol. 50, no. 7, pp. 1535-1548, 2012.

[51] J. H. Saleh, E. A. Saltmarsh, F. M. Favarò, and L. Brevault, "Accident precursors, near misses, and warning signs: critical review and formal definitions within the framework of Discrete Event Systems," Reliability Engineering \& System Safety, vol. 114, no. 1, pp. 148-154, 2013. 


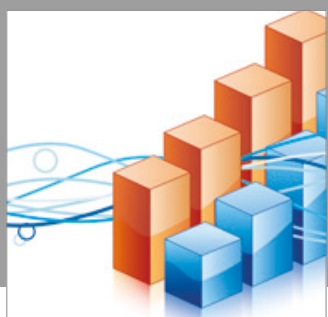

Advances in

Operations Research

vatersals

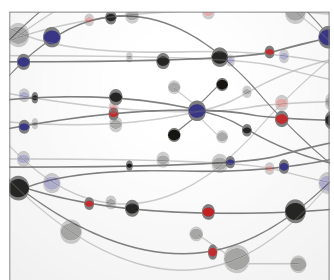

\section{The Scientific} World Journal
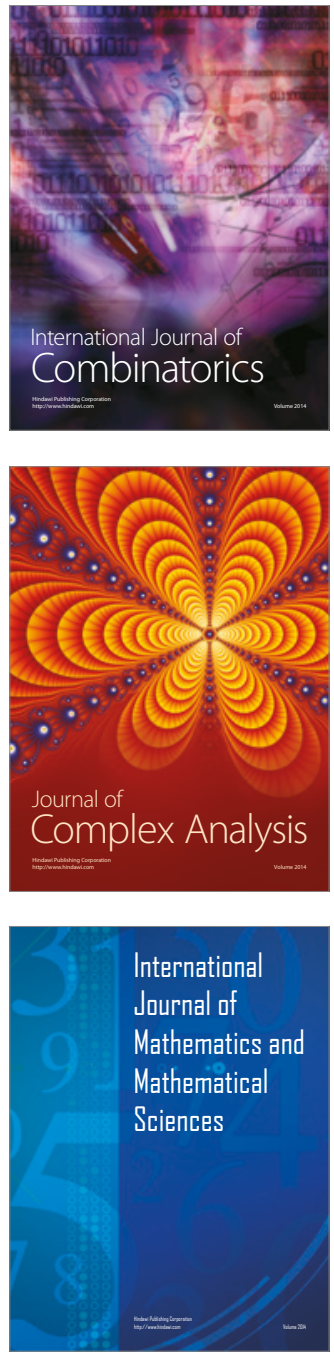
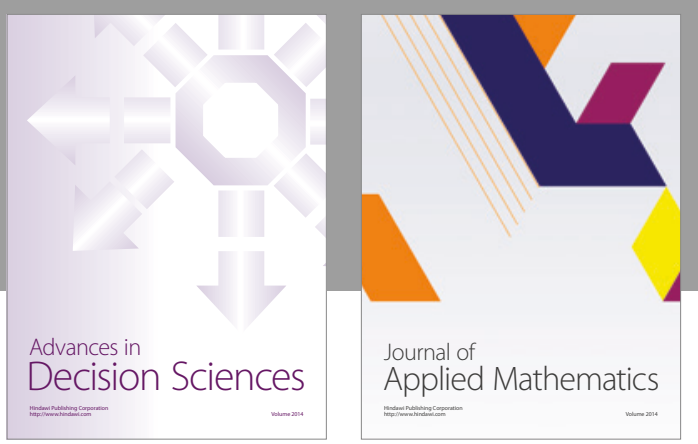

Algebra

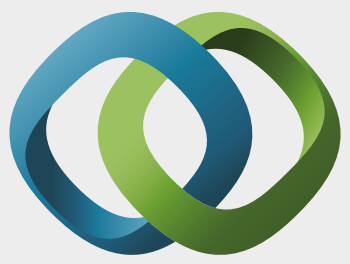

\section{Hindawi}

Submit your manuscripts at

https://www.hindawi.com
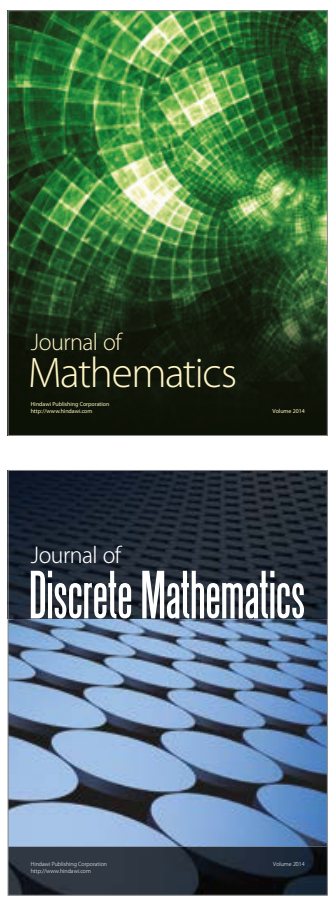

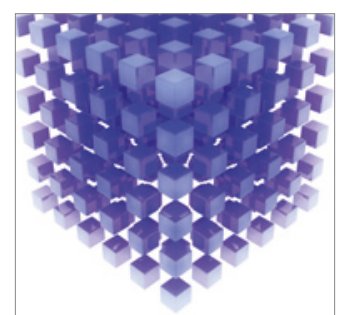

Mathematical Problems in Engineering
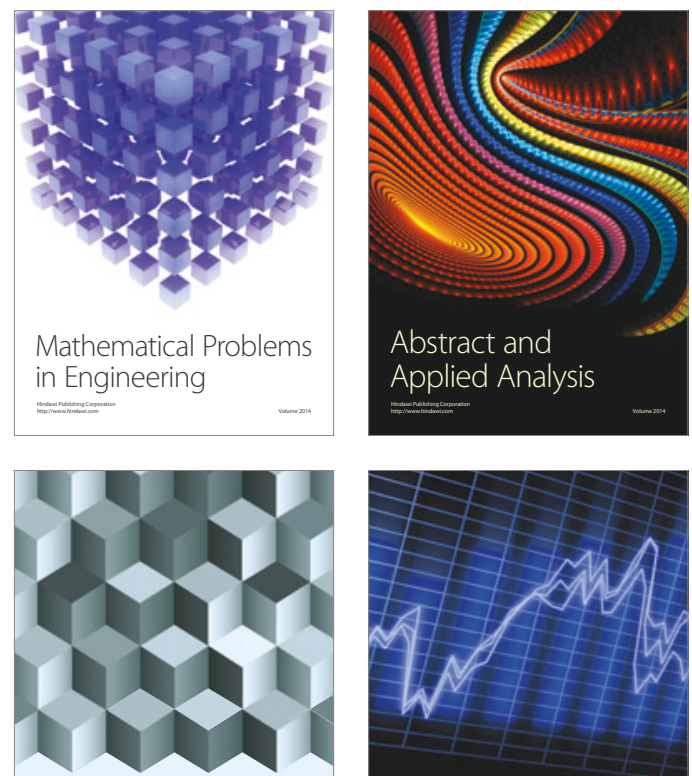

Journal of

Function Spaces

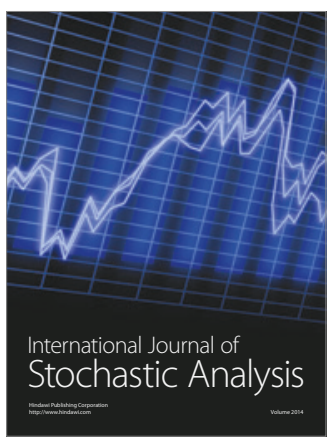

Probability and Statistics
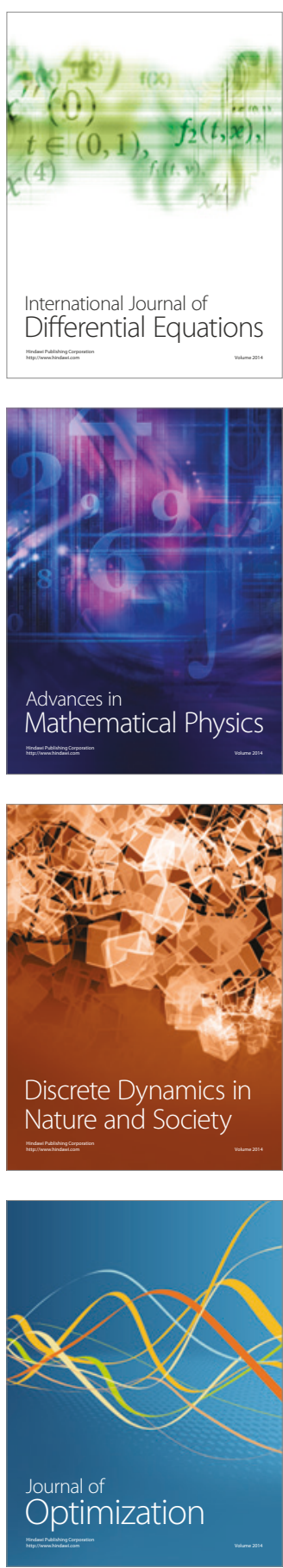